\title{
Robot-guided pediatric stereoelectroencephalography: single-institution experience
}

\author{
Allen L. Ho, MD, ${ }^{1}$ Yagmur Muftuoglu, PhD, ${ }^{1}$ Arjun V. Pendharkar, MD, ${ }^{1}$ Eric S. Sussman, MD, ${ }^{1}$ \\ Brenda E. Porter, MD, PhD, ${ }^{2,3}$ Casey H. Halpern, MD,' and Gerald A. Grant, MD ${ }^{1,4}$ \\ Departments of ${ }^{1}$ Neurosurgery and ${ }^{2}$ Neurology, Stanford University School of Medicine, Stanford; and Divisions of ${ }^{3}$ Pediatric \\ Neurology and ${ }^{4}$ Pediatric Neurosurgery, Lucile Packard Children's Hospital Stanford, California
}

\begin{abstract}
OBJECTIVE Stereoelectroencephalography (SEEG) has increased in popularity for localization of epileptogenic zones in drug-resistant epilepsy because safety, accuracy, and efficacy have been well established in both adult and pediatric populations. Development of robot-guidance technology has greatly enhanced the efficiency of this procedure, without sacrificing safety or precision. To date there have been very limited reports of the use of this new technology in children. The authors present their initial experience using the ROSA platform for robot-guided SEEG in a pediatric population.

METHODS Between February 2016 and October 2017, 20 consecutive patients underwent robot-guided SEEG with the ROSA robotic guidance platform as part of ongoing seizure localization and workup for medically refractory epilepsy of several different etiologies. Medical and surgical history, imaging and trajectory plans, as well as operative records were analyzed retrospectively for surgical accuracy, efficiency, safety, and epilepsy outcomes.

RESULTS A total of 222 leads were placed in 20 patients, with an average of 11.1 leads per patient. The mean total case time $( \pm$ SD) was 297.95 ( \pm 52.96$)$ minutes and the mean operating time per lead was 10.98 minutes/lead, with improvements in total (33.36 minutes/lead vs 21.76 minutes/lead) and operative (13.84 minutes/lead vs 7.06 minutes/lead) case times/lead over the course of the study. The mean radial error was 1.75 ( $\pm 0.94 \mathrm{~mm}$ ). Clinically useful data were obtained from SEEG in $95 \%$ of cases, and epilepsy surgery was indicated and performed in $95 \%$ of patients. In patients who underwent definitive epilepsy surgery with at least a 3-month follow-up, 50\% achieved an Engel class I result (seizure freedom). There were no postoperative complications associated with SEEG placement and monitoring.

CONCLUSIONS In this study, the authors demonstrate that rapid adoption of robot-guided SEEG is possible even at a SEEG-naïve institution, with minimal learning curve. Use of robot guidance for SEEG can lead to significantly decreased operating times while maintaining safety, the overall goals of identification of epileptogenic zones, and improved epilepsy outcomes.

https://thejns.org/doi/abs/10.3171/2018.5.PEDS17718
\end{abstract}

KEYWORDS robotic guidance; robot; stereoelectroencephalography; SEEG; pediatric epilepsy; pediatric SEEG

$\mathrm{S}$ TEREOELECTROENCEPHALOGRAPHY (SEEG) allows for direct electrical recording from cerebral structures to provide high-resolution localization of epileptogenic zones. Its use in drug-resistant epilepsy evaluation and treatment has increased because safety, accuracy, and efficacy have been well established in both adult ${ }^{5}$ and pediatric populations. ${ }^{7,8,11}$ Development of robot-guidance technology has greatly enhanced the efficiency of this procedure, allowing for placement of a greater number of depth electrodes in a shorter amount of time, without sacrificing safety or precision. ${ }^{10,18}$ The ROSA robot guidance platform (ZimmerBiomet/MedTech) was designed specifically for cranial stereotaxy and consists of a robot arm with $6 \mathrm{df}$ attached to a mobile floor-fixed base unit. The base unit attaches directly to the cranial head clamp, and patient imaging registration is completed via skull fiducial or laser registration., ${ }^{2,14}$ Whereas European centers have been using the ROSA robotic platform to plan SEEG for quite some time, experience in centers in the United States and North America is relatively recent. ${ }^{5}$

ABBREVIATIONS LITT = laser interstitial thermal therapy; RNS = responsive neurostimulation; SEEG = stereoelectroencephalography.

SUBMITTED December 16, 2017. ACCEPTED May 10, 2018.

INCLUDE WHEN CITING Published online August 17, 2018; DOI: 10.3171/2018.5.PEDS17718. 
Despite the potential advantages of robot guidance for stereotaxy in SEEG, to date there have been very limited reports of use of this new technology in children, ${ }^{1,9,15}$ with no North American pediatric studies dedicated solely to SEEG. In this study, we describe our initial experience using the ROSA platform for robot-guided SEEG in a pediatric population, and report on operative efficiency, stereotactic accuracy, and epilepsy outcomes.

\section{Methods}

Between February 2016 and October 2017, 20 consecutive pediatric patients underwent robot-guided SEEG as part of ongoing seizure localization and work-up for medically refractory epilepsy of several different etiologies (Table 1). All patients underwent multidisciplinary review by an institutional epilepsy board prior to consideration for SEEG. This study was performed at Lucile Packard Children's Hospital Stanford with approval from the Stanford University Internal Review Board.

\section{Robot-Guided SEEG}

All surgical treatment plans were devised by the senior author (G.A.G.) and the relevant multidisciplinary team. Consensus zones of interest were decided upon in a multidisciplinary epilepsy conference in which seizure history and semiology, and all available phase I video EEG, imaging (including interictal FDG-PET and magnetoencephalography [MEG] in some instances), and other diagnostic data were reviewed. A contrast-enhanced, volumetric MRI sequence of the entire cranial vault with specific T1-weighted, 3D Ultrafast Gradient Echo (BRAVO) sequences is obtained using minimum echo time and repetition time for vascular enhancement and background parenchyma suppression to visualize cortical and subcortical anatomy as well as critical vasculature prior to surgery. A volumetric CT scan is also obtained for superficial surface anatomy for facial registration purposes. Using the manufacturer's ROSA planning software (version 2.5.8), the MRI and CT scans are loaded and fused, then the trajectories for all planned electrodes are prepared. The trajectories were selected to maximize sampling from deep cortical and subcortical areas within the zones of interest. Broad interrogation of possible involved networks based on seizure semiology, video EEG findings, and other workup is prudent, with more directed sampling around zones of interest. Strategic electrode placement completely surrounding zones of interest can allow for clear spatial localization of both seizure onset and direction of propagation. Care was taken in selection of dural entry points and trajectories to avoid traversing any large or small vessels. There was also a low threshold to proceed with bilateral monitoring if there was any question about laterality of onsets. Planning sessions could be quite time-consuming, requiring up to several hours with a greater number of planned trajectories, but every attempt was made to optimize the clarity of SEEG data in order to achieve adequate seizure onset localization. Final trajectory plans were then reviewed by the entire epilepsy team prior to surgery (Fig. 1).

On the day of surgery patients are taken to the operating
TABLE 1. General demographic data in 20 patients with epilepsy who underwent SEEG

\begin{tabular}{lc}
\hline \multicolumn{1}{c}{ Characteristic } & Value \\
\hline Mean age in yrs, \pm SD & $10.9 \pm 5.8$ \\
\hline Male sex & $65 \%(13 / 20)$ \\
\hline Seizure etiology & \\
\hline Nonlesional & 9 \\
\hline Tuberous sclerosis & 3 \\
\hline Encephalomalacia w/ cystic lesion & 2 \\
\hline Benign temporal lesions & 1 \\
\hline Cortical dysplasia & 1 \\
\hline Hemimegalencephaly &
\end{tabular}

room and, under general anesthesia, they are positioned either supine or laterally in Mayfield cranial fixation. The ROSA 2.5 platform base is attached to the headframe, and the robotic arm is used for semiautomatic laser-based facial recognition to register the patient to the imaging. After sterile preparation, draping, and surgical timeout, the procedure begins with the robotic arm moving to each planned trajectory. A stab incision is made at the entry point and a 2-mm-diameter handheld drill is used to create twist drill holes with guidance from the robotic arm. Anchor bolts (Adtech) are firmly screwed into each hole, and a small durotomy is made with an insulated dural perforator and low-powered monopolar cautery. Premeasured electrodes (Epilepsy LTM depth electrodes; Adtech) are then inserted into each hole under robotic arm guidance down to target depth. Electrodes are then secured with polyurethane bolt caps before moving on to the next target. After all electrodes are secured, Xeroform gauze is placed around each bolt and standard head-wrap and netting is applied. Patients remain intubated and are transferred to the CT scanner.

Postoperative volumetric noncontrast 3D CT scans are performed immediately to confirm electrode positioning and to ensure that there is no intracranial hemorrhage or other surgical complication prior to extubation. Patients are monitored clinically and electrographically in the epilepsy monitoring unit for seizure events over the course of several days to a week. Once sufficient data for epilepsy localization are obtained, the electrodes are removed in the operating room under monitored anesthesia care (MAC) and the patient is discharged home either on the same day or the following one. Another multidisciplinary discussion determines the treatment plan.

\section{Data Collection}

Medical and surgical history, imaging, and trajectory plans, as well as operative records were analyzed retrospectively for surgical accuracy, efficiency, safety, and epilepsy outcomes. Patient demographic and clinical data were collected. Complications related to the procedure and postoperative seizure outcomes, including Engel class when available, were also collected. Engel class was determined for patients who underwent resection with at least 3 months of postoperative follow-up. Furthermore, oper- 

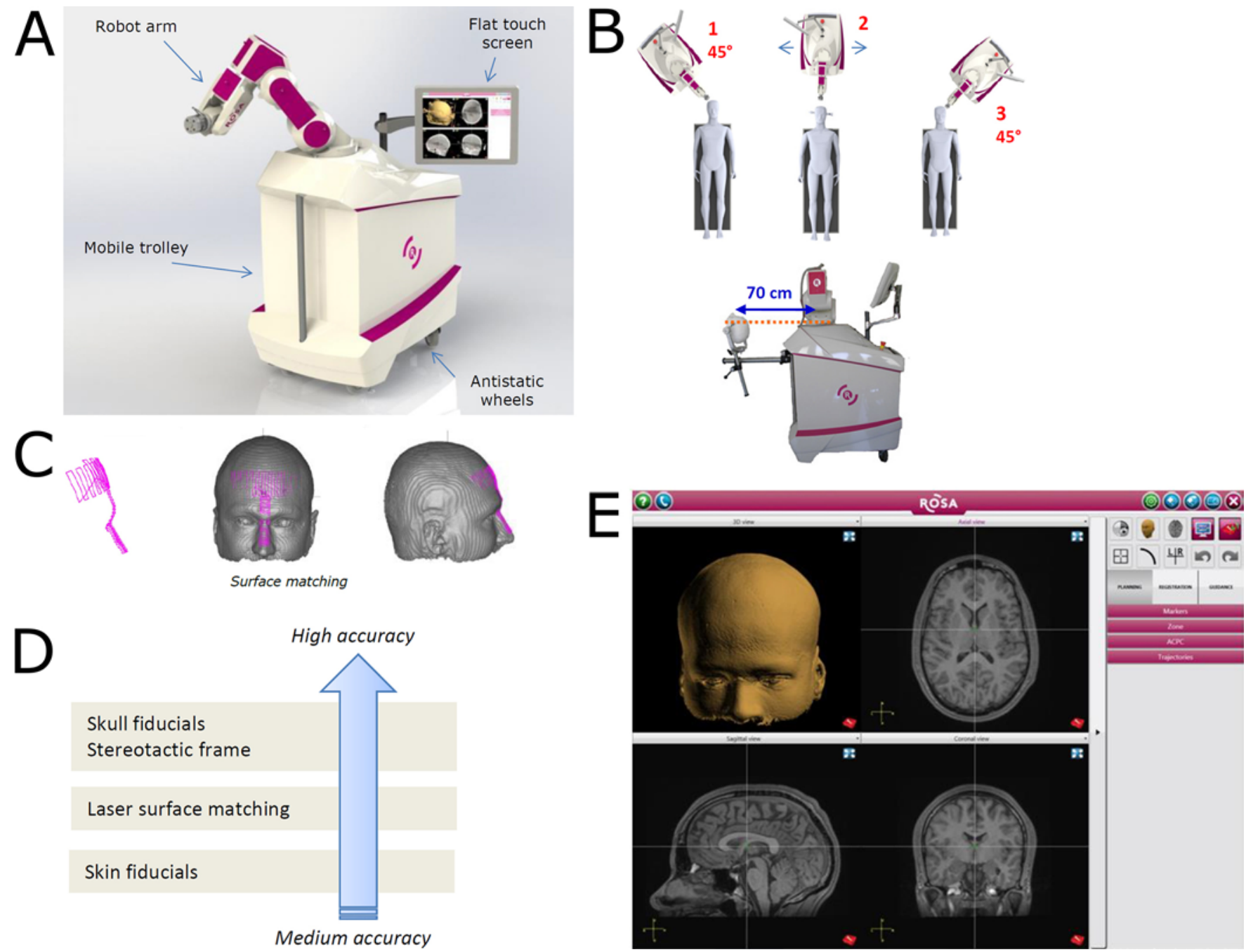

FIG. 1. Robotic-guidance platform schematic. A: The ROSA robot consists of a robot arm with $6 \mathrm{df}$ attached to a mobile floorfixed base unit. B: The base unit attaches directly to the cranial head clamp and platform positioning is critical and dependent on planned location of SEEG electrodes. C: The robot arm, which can be used for semiautomatic laser-based facial recognition, is used to register the patient to the imaging. D: Accuracy of registration increases from skin-placed adhesive fiducials, to laser facial surface matching, to implanted skull fiducials and placement of stereotactic frame. E: Working screen view of the manufacturer's ROSA planning software that is used for image fusion and trajectory planning. Figure is available in color online only.

ating times, number of electrodes and their location, and placement error were recorded and analyzed. Case times were calculated as absolute values, as well as time per lead whenever appropriate. Total case time was defined as time from anesthesia induction to extubation. Operating time was defined as time from incision to closure. Positioning time was defined as time from anesthesia handoff to time of incision, and includes the laser registration. Preoperative planning MR images used with trajectory plans were merged with postoperative CT scans obtained with electrodes in place to assess the accuracy of the electrode position in relation to preoperative planned trajectories. 2D radial errors were defined as the distance between the planned target and the intersection of the device axis with the target plane, and depth errors were defined as the error from the device tip to the intersection of the device axis with the target plane ${ }^{14}$ (Fig. 2). Target point error was calculated as the absolute distance between the electrode tip and target point. All errors were calculated within the ROSA planning software.

\section{Results \\ Patient Demographics}

Demographic data for our cohort are reported in Table 1 . The mean age of patients in the cohort was 10.9 years, with a range of 2-20 years of age. The number of males was $13 / 20$ in our cohort. There were 9 nonlesional cases, and the distribution of other underlying structural etiology is detailed.

\section{Case Times}

The mean total case time $( \pm$ SD) was $297.95( \pm 52.96)$ minutes, and the mean total operating and positioning times 


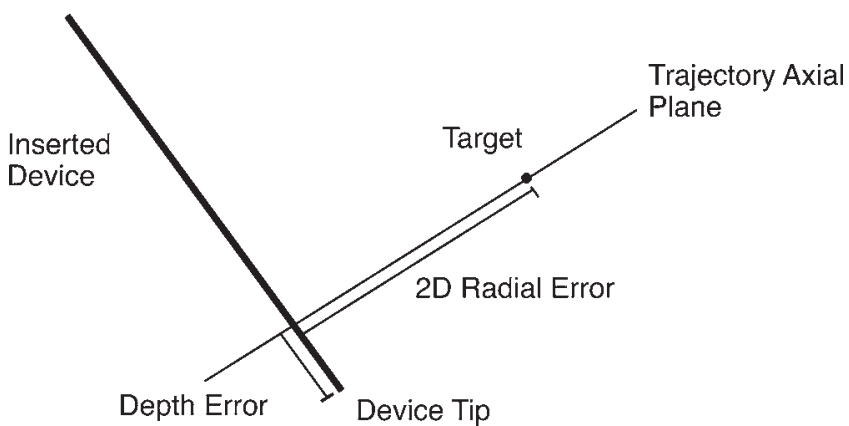

FIG. 2. Schematic showing trajectory plane errors. The $2 \mathrm{D}$ radial error is the distance between the planned target and the intersection of the device axis with the target plane, and was measured in millimeters. The depth error is the distance from the device tip to the intersection of the device axis with the target plane, and was measured in millimeters. Reprinted with permission from Ho AL et al: Improved operative efficiency using a real-time MRI-guided stereotactic platform for laser amygdalohippocampotomy. J Neurosurg 128:1165-1172, 2018.

were $117.15( \pm 47.96)$ minutes and $114.75( \pm 43.65)$ minutes, respectively. In looking at trends in total case times over the course of our study, there was a slight trend toward decreasing total and operative case times (Fig. 3). When looking at case times per lead, there are clear decreases in both total case time per lead (mean 27.67 minutes/lead) as well as operative case time per lead (mean 10.98 minutes/lead). The averages for the first and last 5 cases in the cohort were 33.36 minutes/lead versus 21.76 minutes/lead for total case time/lead and 13.84 minutes/lead versus 7.06 minutes/lead for operative case time/lead (Fig. 4). However, positioning case times remained relatively constant throughout (first 5 cases averaged 10.67 minutes/lead vs an average for the last 5 of 9.96 minutes/lead).

\section{Surgical Data and Accuracy}

A total of 222 leads were placed with an average of 11.1 $( \pm 2.4)$ and a range of 6-16 leads per patient. Of these, 104 were frontal, 73 temporal, 29 parietal, and 16 occipital. There were no electrode failures in our study. Accuracy data can be found in Table 2. The mean radial error was $1.75( \pm 0.94 \mathrm{~mm})$, with a range of $0.91-3.62 \mathrm{~mm}$. The mean depth error was $2.82( \pm 1.1 \mathrm{~mm})$, with a range of 0.9-4.02 $\mathrm{mm}$. The mean target point error was $3.39( \pm 1.078 \mathrm{~mm})$, with a range of $1.78-4.98 \mathrm{~mm}$.

\section{Complications}

There were no intracranial hemorrhages noted on postoperative CT scans, and no surgical site infections or any other postoperative complications associated with the SEEG in any of our patients.

\section{Epilepsy Outcomes}

All patients undergoing robot-guided SEEG had seizures that had been successfully captured on SEEG. This was after an average duration of monitoring of $5( \pm 3.2)$ days and an overall length of stay of $5.85( \pm 3.27)$ days. Clinically useful data were obtained from these SEEG studies in $95 \%$ of cases, and epilepsy surgery was com-

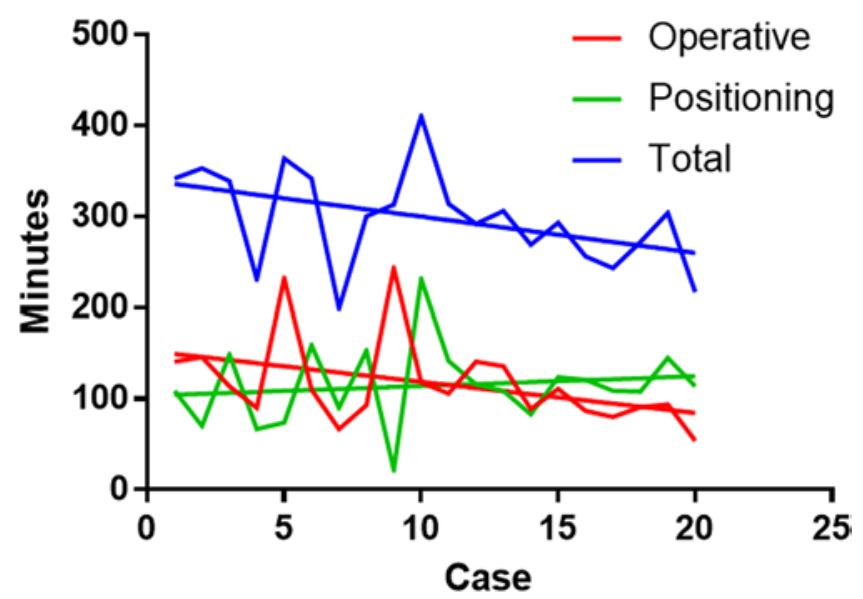

FIG. 3. Graph showing case times. Operative, positioning, and total case times demonstrate decreased total and operative case times over the course of the study, with relatively constant positioning case times over the course of the study. Figure is available in color online only.

pleted or is being proposed for $95 \%$ of patients. In 1 patient, SEEG revealed bilateral, more generalized seizure onsets without clear foci that were believed to be not amenable to further localization. All other patients underwent or were offered a subsequent procedure to treat the refractory epilepsy based on the SEEG data obtained. Fourteen patients underwent or were offered a resective surgery, 2 patients underwent responsive neurostimulation (RNS), 2 patients have been offered RNS, and 1 patient had laser interstitial thermal therapy (LITT). There was an average postoperative follow-up of 7.92 months. In patients who underwent definitive epilepsy surgery with at least 3 months of follow-up $(\mathrm{n}=12), 50 \%$ achieved an Engel class I result (seizure freedom), 25\% had Engel class III (some seizure reduction), and $25 \%$ had Engel class IV (no improvement in seizures) (Table 3 ).

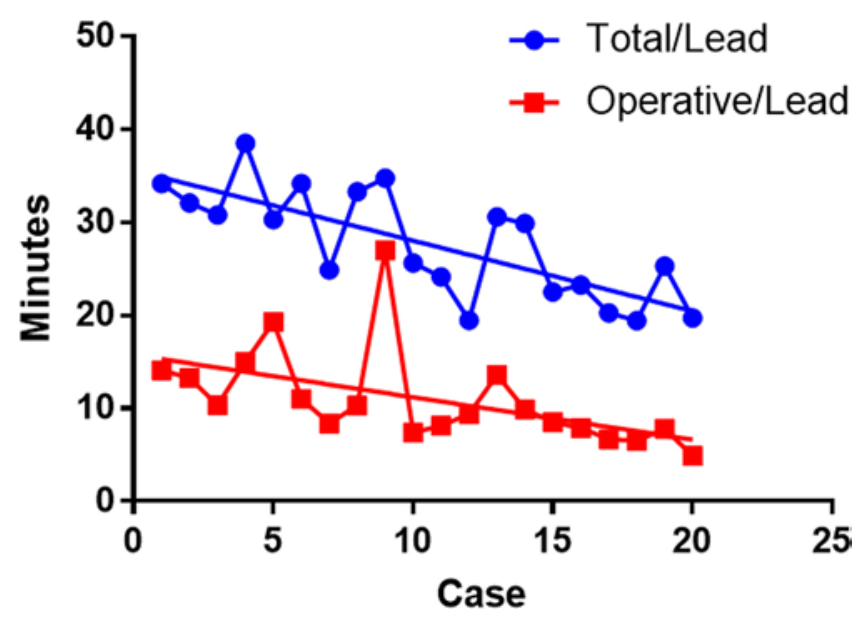

FIG. 4. Graph showing case times/lead. Total and operative case times/ lead demonstrate a trend of decreasing total and operative case time/ lead over the course of the study, underscoring efficiency gains made as familiarity with the robotic-guidance platform developed over time. Figure is available in color online only. 
TABLE 2. Surgical outcomes in 20 patients with epilepsy who underwent SEEG

\begin{tabular}{cc}
\hline Surgical Outcomes & Value \\
\hline Mean no. of leads/patient & $11.1 \pm 2.4$ \\
\hline Total leads implanted & 222 \\
\hline Lead location & \\
\hline Bilateral & $9 / 20$ \\
\hline Frontal & 104 \\
\hline Temporal & 73 \\
\hline Parietal & 29 \\
\hline Occipital & 16 \\
\hline Mean time (min) & $297.95 \pm 52.96$ \\
\hline Total case time & $27.67 \pm 5.91$ \\
\hline Total case time/lead & $10.98 \pm 5.12$ \\
\hline Op time/lead & $114.75 \pm 43.65$ \\
\hline Total positioning time & \\
\hline Mean errors (mm) & $2.82 \pm 1.1$ \\
\hline Depth error & $1.75 \pm 0.94$ \\
\hline Radial error & $3.39 \pm 1.078$ \\
\hline Target point error &
\end{tabular}

Unless otherwise indicated, values are expressed as the mean \pm SD.

\section{Discussion}

Stereoelectroencephalography has been rediscovered and has seen increasing popularity over the last decade, ${ }^{3}$ much of this due to advancements made in neuroimaging and image-guided stereotaxy that have given surgeons more precision and accuracy in placement of depth electrodes in a targeted fashion..$^{5}$ The advent of robotic stereotaxy has only allowed for increased efficiency via shorter time of implantation with preservation of accuracy and safety. ${ }^{10}$ Early data also demonstrated that increased efficiency leads to higher-resolution electrophysiological and anatomical definition of epileptogenic zones that may lead to improved seizure outcomes, especially in patients with difficult-to-localize seizures., ${ }^{4}, 18$ However, long-term follow-up is necessary to confirm the durability of these outcomes. Whereas robot-guided SEEG has been studied in adults, there remain few dedicated pediatric experiences published in the literature. We present our initial series of 20 pediatric patients undergoing robot-guided SEEG; our findings demonstrate comparable operative efficiency, accuracy, and safety as well as epilepsy outcomes compared to previously published robot-guided and nonrobot-guided SEEG series. Our work demonstrates that rapid adoption of this technology and technique is possible even at a SEEG-naïve institution, with a minimal learning curve.

\section{Operative Efficiency With SEEG}

In terms of operative efficiency, in our initial 20 patients we placed a total of 222 electrodes, with a mean of $11.1( \pm 2.4)$ electrodes per patient. Our mean total operating time was $117.15( \pm 47.96)$ minutes, with a mean 10.98 $( \pm 5.12)$ minutes/lead, which is comparable to the 2 prior pediatric case series (10.9 minutes/electrode in Miller et
TABLE 3. Epilepsy outcomes in 20 patients who underwent SEEG

\begin{tabular}{cc}
\hline \multicolumn{1}{c}{ SEEG \& Epilepsy Outcomes } & Value \\
\hline Seizure capture & $100 \%$ \\
\hline Duration of monitoring (days) $^{*}$ & $5 \pm 3.2$ \\
\hline Length of stay (days) $^{*}$ Epileptogenic zone localization & $5.85 \pm 3.27$ \\
\hline Completed/planned epilepsy surgery & $95 \%$ \\
\hline Resection & $95 \%$ \\
\hline RNS & 14 \\
\hline LITT & 4 \\
\hline Follow-up (mos) & 1 \\
\hline Engel class & $7.92 \pm 5.79$ \\
\hline I & $50 \%$ \\
\hline II & $0 \%$ \\
\hline III & $25 \%$ \\
\hline IV & $25 \%$ \\
\hline
\end{tabular}

* Values expressed as the mean \pm SD.

al.; 19.09 minutes/electrode extrapolated from the mean total operating time/mean number of electrodes per patient in De Benedictis et al.). ${ }^{915}$ Our operating time is also on par with the largest adult series, with case times from Cleveland Clinic that reported a mean operating time of 130 minutes, mean 12.5 electrodes per patient, and an extrapolated mean 10.4 minutes/electrode. It should also be noted that although our positioning time remained relatively constant throughout the series (Fig. 4), our operating times continued to drop such that the mean operating time/electrode for the last 10 and the last 5 patients was 8.25 and 6.76 minutes, respectively. This indicates that although the positioning and setup of the case may seem cumbersome given the need to attach the robot base to the headframe and register the patient, there is no significant learning curve with this process. Furthermore, relative efficiency can be obtained within a small number of cases when compared to the most experienced robotic centers, especially when compared to conventional nonrobot-guided techniques.

\section{Target Point Accuracy in SEEG}

Accuracy measurement in SEEG has been difficult to standardize across the literature due to heterogeneity in techniques, reporting, and quality of the evidence. A recently published meta-analysis that aggregated all published reports of SEEG found combined mean target point errors of $2.89 \mathrm{~mm}$ (95\% CI 2.34-3.44 mm), $1.93 \mathrm{~mm}$ (95\% CI $1.05-2.81 \mathrm{~mm})$, and $1.17 \mathrm{~mm}(95 \%$ CI $1.66-1.75 \mathrm{~mm})$ for frameless, frame-based, and robotic guidance systems, respectively. ${ }^{21}$ Our target point error is higher than typically reported for robotic guidance systems at $3.39 \pm 1.078$ $\mathrm{mm}$, and is more similar to what is seen for frameless systems. Target point accuracy can be affected by a multitude of factors including trajectory angle (even when the entry point is accurate), electrode deflection by dura mater or brain, and electrode rigidity, as well as registration and intraoperative trajectory modifications. We believe that the 
last two factors probably play the largest role in our results. With the exception of 1 patient with occipital localization who was placed prone and who received skull fiducials, we used laser facial recognition without skull fiducials in our patients, given decreased tolerance in pediatric patients to placement of skull fiducials, and to obviate the need for additional radiation from a pre- or intraoperative registration CT scan. However, this approach can theoretically lead to less precise registration that can negatively impact accuracy. ${ }^{13}$

There are several registration approaches available with the ROSA, each with its specific considerations for pediatric patients. Current ROSA models allow for point-based frame registration that is similar to point-based skull fiducial registration in that the frame is registered to the robot via touch-point registration along the frame. A forthcoming ROSA model includes a software module for automated imaging-based identification of the frame within the registration software (http://www.medtech.fr/en/rosabrain). In a comparative study, frame-based with intraoperative CT registration, skull fiducial-based with intraoperative CT registration, and laser facial recognition-based registration have yielded errors of $0.81 \mathrm{~mm}, 0.7 \mathrm{~mm}$, and $1.22 \mathrm{~mm}$, respectively. ${ }^{13}$ A more recent study comparing skull fiducial-based and laser facial recognition-based registration techniques without intraoperative $\mathrm{CT}$ revealed no significant difference between the two techniques (3.9 $\mathrm{mm}$ vs $4.5 \mathrm{~mm}, \mathrm{p}=0.19){ }^{2}$ Skull fiducials and frame placements may need to take place preoperatively and may not be well tolerated by younger patients. Intraoperative registration scans can be done to avoid awake placement of more rigid registration options, but these approaches have the disadvantage of adding additional anesthesia time and additional radiation from the registration CT scan. Finally, there is some evidence to suggest that comparable registration accuracy could be achieved with laser surface registration performed with preoperative MRI scans only, which could further streamline the workflow of the procedure as well as limit radiation exposure. ${ }^{20}$ These approaches all warrant further study to continue to optimize registration workflow for pediatric patients undergoing SEEG.

Finally, there were sometimes significant modifications made to planned trajectories intraoperatively due to troubleshooting. For example, if the planned entry point was situated in bone too thin to hold our guide bolts, the trajectory may have been altered to be situated in more robust skull, but was designed to conform to the same epileptogenic zone-monitoring purposes. This was not accounted for in our accuracy measurements because we used preoperative trajectory plans for our calculations. It should also be noted that our higher target point error did not seem to impact our complications or overall seizure outcomes.

\section{Complications of SEEG}

We did not note any postoperative infection in these patients. We did routinely shave small $1-\mathrm{cm}$ areas of hair over the proposed entry points for all electrodes prior to the surgical preparation, but it is not known if that played any role in the exceedingly low infection rate. We also removed all electrodes in the operating room under sterile conditions. This result is similar to low infection rates seen in other re- cent pediatric SEEG series, ${ }^{7,8,11}$ and compares favorably to infection rates reported with subdural electrode monitoring in children, which range from $0 \%$ to $8.6 \% .^{12,17,19}$

The T1-weighted, 3D Ultrafast Gradient Echo (BRAVO) protocol at our institution provides exquisite detail of the large and small vessels in the brain. We believe that this resolution is critical to the planning and safety of the SEEG placement. We did not see any hemorrhage following SEEG placement on CT in our series. We did not routinely repeat imaging in children after SEEG removal prior to discharge. We attest that this low hemorrhage rate is due to the routine systematic double-check by the attending surgeon and the fellow or resident as well as the exquisite detail we get with our imaging protocol. Again, this is similar to the low intracranial hemorrhage rates reported in other recent pediatric SEEG series, $, 8,11$ and compares favorably to intracranial hemorrhage rates reported with subdural electrode monitoring in children, which range from $0 \%$ to $23 \%$. $12,17,19$

\section{Patient Outcome Using SEEG}

Many centers have demonstrated improved ability to identify epileptogenic zones and improved seizure outcomes in a safe manner with SEEG. ${ }^{3,16}$ Thus, this technique is being widely adopted by more and more epilepsy centers as a crucial step in the comprehensive methodology for identification of epileptogenic zones in both lesional and nonlesional medically refractory epilepsy. ${ }^{5,15}$ This is particularly relevant in children for whom early identification and treatment of epileptogenic zones can have a lifelong effect. Our seizure outcomes were similar to those in previously published series, with epileptogenic zone localization in $90 \%$ of patients and with $90 \%$ of patients either undergoing or at least offered epilepsy surgery in some form (resection, LITT, or RNS), and with 50\% of patients achieving an Engel class I outcome of seizure freedom with a mean follow-up of 7.92 months. Thus, this procedure has a clear role in obtaining meaningful electrographic data that lead to more surgical options and improved epilepsy outcomes in pediatric patients with medically refractory epilepsy, especially in nonlesional cases. It should be noted that the average duration of monitoring was shorter, at $5( \pm 3.2)$ days, compared to adult series of 8 days in the Cleveland Clinic series, which is probably due to higher seizure frequency in pediatric patients, although less patient tolerance in the pediatric population of longer periods of SEEG monitoring cannot be ruled out. ${ }^{10}$

\section{Technical Pearls}

In terms of trajectory planning, orthogonal trajectories can allow for ease of interpretation and accuracy. In the pediatric population, it is more challenging to develop a naming convention for the SEEG electrodes. In many patients the disease is nonlesional or extratemporal, and also placement is customized in addition to more standard locations to target such as mesial temporal, orbitofrontal, and anterior cingulate. High-resolution vascular imaging to facilitate avoidance of cortical arteries and veins is critical, although no consensus modality has been established. Careful electrode selection in terms of density and lead length must be based on planned trajectories to maximize 
parenchymal recording. Broad interrogation of possible involved networks based on seizure semiology, video EEG, and other workup is prudent, with more directed sampling around zones of interest. The SEEG approach allows for repeated higher-density sampling of zones of interest, or cortical mapping with grids and strips of eloquent cortex as a second procedure if necessary.

An intention to treat approach to depth trajectory planning may be appropriate for certain patients in whom trajectories and anchoring bolts can be used for laser catheter placement for ablation, or depth electrodes can be left in place to guide resection. We also have proceeded directly to an RNS device after SEEG. This approach extends to anchor bolt selection as well because possible laser catheter placement may necessitate larger-diameter anchoring bolts. Considerations can also be given for possible stimulation mapping, white matter mapping, functional mapping, or even possible depth electrode-mediated radiofrequency ablation of seizure foci with initial trajectory planning. ${ }^{6}$ Trajectory order and preplacement robot arm range of motion verification can help avoid difficulties once depth electrode placement has begun. There is a limit to the range of motion of the robot arm and, although a planned trajectory may be able to be achieved by the arm, the working distance of the arm may be limited, which can make drilling less precise or impossible. There are also theoretical concerns for brain molding from SEEG placement that can confer some stereotactic error, so placement of longer and deeper trajectories first may minimize these errors.

With regard to anchoring bolts, there are several points that must be considered in relation to the skull thickness, especially in pediatric patients, and tangential trajectory angulation that can help avoid loose or inaccurate anchor placement. Preoperatively, great care should be taken to appropriately space entry points such that anchor screws with approximately $1 \mathrm{~cm}$ (or more) of extracranial clearance and tangential angulation do not obstruct each other. Intracranial clearance between trajectories of at least $1 \mathrm{~cm}$ can also help ensure no intracranial lead collisions. We have narrowed this gap when necessary to avoid critical vasculature, particularly in the temporal lobe. We also hold off on placing the securing cap until we have placed the adjacent bolt and electrode, because the cap is wider than the bolt. The anchoring bolt will help maintain the electrode trajectory, so rigid fixation is critical for maintenance of accuracy. Skull thickness at the entry point is important for anchor bolt selection, and the bolt's length must be appropriately sized to match the skull and soft-tissue thickness as closely as possible to not allow for toggle and fracture of thinner skull areas from torque on the screw anchor. In our experience, the squamous temporal bone was always the thinnest bone, and any bone thickness less than $2 \mathrm{~mm}$ in this area was cause for concern.

In contrast to adult patients, in whom thick temporalis muscle necessitates thicker anchoring bolts, smaller anchoring bolts are often sufficient in children. There were occasional anchor screws in this area only that were thought to be too loose after placement and that necessitated rescue screw placement or intraoperative replanning of trajectory to drill a new pilot hole. Sometimes more tangential trajectories placed through thicker areas of skull allowed for similar anatomical coverage, but with increased length of fixation and improved purchase of anchor bolts in the skull. A "bottom-up" oblique trajectory is also sometimes helpful in thicker inferior temporal bone. Drilling through both outer and inner tables of the skull along with sharp opening of the dura and pia ensure that these structures do not interfere with electrode placement. At the close of our operation, the head is completely wrapped extensively with 2 layers of Kerlix gauze dressing that will only be changed during the patient's admission if it unravels. With this technique and with the meticulous care of the dressing and electrodes provided by our epilepsy monitoring unit technicians, we have not had any anchor bolts loosen or dislodge during monitoring.

\section{Limitations of the Study}

The main limitation to our study is the fact that it is a retrospective, single-armed study. This was due to the fact that SEEG was not previously performed at our pediatric hospital, and so a comparison cohort of patients who underwent nonrobot-guided SEEG was not available for analysis. However, as detailed above, we were able to compare our surgical results and epilepsy outcomes to the published results of the most experienced SEEG and robotic SEEG centers, and our study was overall on par. The follow-up interval for epilepsy outcomes was at least 3 months, with a mean of 7.92 months, which is inadequate to draw conclusions regarding long-term epilepsy outcomes. Therefore, further study with more long-term follow-up results would be informative.

\section{Conclusions}

Our initial experience in the adoption of robot-guided SEEG has demonstrated comparable operative efficiency, accuracy, and safety as well as epilepsy outcomes compared to previously published robot-guided and nonrobotguided SEEG series. Based on our study, rapid adoption of this technology and technique is possible even at a SEEGnaïve institution, with a minimal learning curve. Use of robot guidance for SEEG can lead to significantly decreased operating times while maintaining safety, the overall goals of identification of epileptogenic zones, and comparable seizure-free outcomes. Ultimately, we strive for seizure freedom and enhanced quality of life by identification of the epileptogenic zone with the greatest possible accuracy and precision and the least possible morbidity.

\section{References}

1. Abel TJ, Varela Osorio R, Amorim-Leite R, Mathieu F, Kahane P, Minotti L, et al: Frameless robot-assisted stereoelectroencephalography in children: technical aspects and comparison with Talairach frame technique. J Neurosurg Pediatr [epub ahead of print April 20, 2018; DOI: 10.3171/2018.1.PEDS17435]

2. Brandmeir NJ, Savaliya S, Rohatgi P, Sather M: The comparative accuracy of the ROSA stereotactic robot across a wide range of clinical applications and registration techniques. J Robot Surg 12:157-163, 2018

3. Cardinale F, Casaceli G, Raneri F, Miller J, Lo Russo G: Implantation of stereoelectroencephalography electrodes: a systematic review. J Clin Neurophysiol 33:490-502, 2016 
4. Cardinale F, Cossu M, Castana L, Casaceli G, Schiariti MP, Miserocchi A, et al: Stereoelectroencephalography: surgical methodology, safety, and stereotactic application accuracy in 500 procedures. Neurosurgery 72:353-366, 2013

5. Chabardes S, Abel TJ, Cardinale F, Kahane P: Commentary: Understanding stereoelectroencephalography: what's next? Neurosurgery 82:E15-E16, 2018

6. Cossu M, Cardinale F, Casaceli G, Castana L, Consales A, D'Orio P, et al: Stereo-EEG-guided radiofrequency thermocoagulations. Epilepsia 58 (Suppl 1):66-72, 2017

7. Cossu M, Cardinale F, Colombo N, Mai R, Nobili L, Sartori I, et al: Stereoelectroencephalography in the presurgical evaluation of children with drug-resistant focal epilepsy. $\mathbf{J}$ Neurosurg 103 (4 Suppl):333-343, 2005

8. Cossu M, Schiariti M, Francione S, Fuschillo D, Gozzo F, Nobili L, et al: Stereoelectroencephalography in the presurgical evaluation of focal epilepsy in infancy and early childhood. J Neurosurg Pediatr 9:290-300, 2012

9. De Benedictis A, Trezza A, Carai A, Genovese E, Procaccini $\mathrm{E}$, Messina R, et al: Robot-assisted procedures in pediatric neurosurgery. Neurosurg Focus 42(5):E7, 2017

10. González-Martínez J, Bulacio J, Thompson S, Gale J, Smithason S, Najm I, et al: Technique, results, and complications related to robot-assisted stereoelectroencephalography. Neurosurgery 78:169-180, 2016

11. Gonzalez-Martinez J, Mullin J, Bulacio J, Gupta A, Enatsu R, Najm I, et al: Stereoelectroencephalography in children and adolescents with difficult-to-localize refractory focal epilepsy. Neurosurgery 75:258-268, 2014

12. Johnston JM Jr, Mangano FT, Ojemann JG, Park TS, Trevathan E, Smyth MD: Complications of invasive subdural electrode monitoring at St. Louis Children's Hospital, 19942005. J Neurosurg 105 (5 Suppl):343-347, 2006

13. Lefranc M, Capel C, Pruvot AS, Fichten A, Desenclos C, Toussaint $\mathrm{P}$, et al: The impact of the reference imaging modality, registration method and intraoperative flat-panel computed tomography on the accuracy of the ROSA ${ }^{\circledR}$ stereotactic robot. Stereotact Funct Neurosurg 92:242-250, 2014

14. Lefranc M, Le Gars D: Robotic implantation of deep brain stimulation leads, assisted by intra-operative, flat-panel CT. Acta Neurochir (Wien) 154:2069-2074, 2012

15. Miller BA, Salehi A, Limbrick DD Jr, Smyth MD: Applications of a robotic stereotactic arm for pediatric epilepsy and neurooncology surgery. J Neurosurg Pediatr 20:364-370, 2017

16. Mullin JP, Shriver M, Alomar S, Najm I, Bulacio J, Chauvel $\mathrm{P}$, et al: Is SEEG safe? A systematic review and meta-analysis of stereo-electroencephalography-related complications. Epilepsia 57:386-401, 2016

17. Önal C, Otsubo H, Araki T, Chitoku S, Ochi A, Weiss S, et al: Complications of invasive subdural grid monitoring in children with epilepsy. J Neurosurg 98:1017-1026, 2003

18. Serletis D, Bulacio J, Bingaman W, Najm I, GonzálezMartínez J: The stereotactic approach for mapping epileptic networks: a prospective study of 200 patients. J Neurosurg 121:1239-1246, 2014

19. Simon SL, Telfeian A, Duhaime AC: Complications of invasive monitoring used in intractable pediatric epilepsy. Pediatr Neurosurg 38:47-52, 2003

20. Spyrantis A, Cattani A, Strzelczyk A, Rosenow F, Seifert V, Freiman TM: Robot-guided stereoelectroencephalography without a computed tomography scan for referencing: analysis of accuracy. Int J Med Robot 14:e1888, 2018

21. Vakharia VN, Sparks R, O'Keeffe AG, Rodionov R, Miserocchi A, McEvoy A, et al: Accuracy of intracranial electrode placement for stereoencephalography: a systematic review and meta-analysis. Epilepsia 58:921-932, 2017

\section{Disclosures}

The authors report no conflict of interest concerning the materials or methods used in this study or the findings specified in this paper.

\section{Author Contributions}

Conception and design: Grant, Ho, Pendharkar, Sussman, Porter, Halpern. Acquisition of data: Ho, Muftuoglu, Pendharkar, Sussman. Analysis and interpretation of data: Grant, Ho, Porter, Halpern. Drafting the article: Ho. Critically revising the article: all authors. Reviewed submitted version of manuscript: Grant, Ho, Pendharkar, Sussman, Porter, Halpern. Approved the final version of the manuscript on behalf of all authors: Grant. Statistical analysis: Ho. Administrative/technical/material support: Grant, Ho, Pendharkar, Sussman, Porter, Halpern. Study supervision: Grant, Ho, Porter, Halpern.

\section{Correspondence}

Gerald A. Grant: Stanford University/Lucile Packard Children's Hospital, Stanford, CA. ggrant2@stanford.edu. 